\title{
A NEW DIAPHRAGMATIC STETHOSCOPE
}

\author{
BY \\ C. R. BURCH AND J. P. P. STOCK \\ From the H. H. Wills Physics Laboratory, Bristol University, and the Stoke-on-Trent Hospital Group
}

Received January 10, 1961

The stethoscope that forms the subject of this paper was designed to give as great a response as possible to the range of frequencies from 250 to 1500 cycles. Standard commercial diaphragmatic stethoscopes are not designed for high sensitivity in this range, yet in clinical auscultation of the heart, these frequencies are often of crucial importance. We therefore decided to disregard previous practice and make a new design for a diaphragmatic stethoscope.

With the new stethoscope, the higher frequency components of normal and abnormal heart sounds and murmurs are heard with much greater clarity. In some patients it has enabled us to hear faint high frequency murmurs and narrow splitting of sounds that were inaudible with an ordinary stethoscope. Detailed measurements, with full electronic instrumentation, of all the properties of its acoustic circuit over a wide range of frequencies, under the condition of use, would almost certainly point the way to further improvement, and we hope that others with the necessary facilities may undertake research in this field.

\section{METHOD}

First, we must decide the frequency response at which to aim. We do not want the maximum possible response at every frequency, for this would overload the ear with low frequencies and so lessen its sensitivity to higher frequencies-for it is known that loud-pitched sounds have this "blanketing" effect. We aim, therefore, at a response increasing with frequency up to, say, 1000 cycles-or possibly even 2000 - and we are, indeed, accustomed to receive most of our verbal information in the range 200 to 2200 cycles (the telephone range). It will be surprising if we succeed in making the stethoscope too sensitive above 2000 cycles.

Even at 1000 cycles, the length of the tube joining the chest-piece to the ears is of the order of a wavelength. We should think, therefore, in terms of guided wave (transmission line) theory and impedance matching. Presumably Nature has arranged that the auditory mechanism includes a meatus that is fairly free of reflections over a band of frequencies around 1000 cycles (the range to which the ear is most sensitive).

The meatus is about $6 \mathrm{~mm}$. in diameter and we should therefore use ear tubes about $6 \mathrm{~mm}$. in diameter, joined by a Y-piece to a single tube $8.5 \mathrm{~mm} .(=6 \sqrt{ } 2 \mathrm{~mm}$. $)$ diameter so as to minimize reflections at the meatus or the Y-piece. If we match the acoustic impedance of the diaphragm unit to that of the chest wall, we shall accept as much energy as possible from the wave reaching the chest surface. Since flesh has a density about 1000 times that of air and sound travels somewhat faster in flesh (like water) than in air, we must make the hole in the back of the diaphragm casing somewhat less than $1 / 1000$ times the area of the diaphragm, if there is to be no reflection at the diaphragm and if the whole energy of the wave is to pass on as a plane wave travelling through the hole. Therefore we choose a hole of $1 \mathrm{~mm}$. diameter for a $45 \mathrm{~mm}$. diameter diaphragm.

If we could make the space behind the diaphragm so small that the radial velocity of the air was at every point about the same as its velocity through the hole, we would have no standing wave and 
hence no unnecessary "dead" space. For a hole of $1 \mathrm{~mm}$. diameter this would imply $0.25 \mathrm{~mm}$. depth behind the diaphragm at the end of the hole and about $0.01 \mathrm{~mm}$. half-way out, decreasing to zero at the edge of the diaphragm. A depth of $0.25 \mathrm{~mm}$. may be practicable but $0.01 \mathrm{~mm}$. certainly is not, since very slight pressure would bring the diaphragm into contact with the chest-piece. Therefore we cannot avoid a dead space and it is the dead space that principally controls the problem of impedance matching between the chest-piece and the $8.5 \mathrm{~mm}$. ear tube. Its volume will be of the order of $0.5 \mathrm{ml}$. and its capacitative impedance at 1000 cycles will be about twelve times smaller than the resistive total impedance that we would like to have behind the diaphragm. We shall, therefore, need to make the $1-8.5 \mathrm{~mm}$. joining piece inductive. Rough calculation shows that a taper some $5 \mathrm{~cm}$. long would give a resonant frequency of the order of 1000 cycles. This would be the frequency of maximum response. (Even at resonance, the resistive component of total impedance is unlikely to be too big.) Resonance may be expected to be fairly flat, because of the resistive loading provided by the ear tube.

A taper reamer of $11^{\circ} 22^{\prime}$ included angle, already available, is used to make the joining tube. The hole is slightly rounded where it intersects with the front face of the chest-piece, which is flat with an $18^{\circ}$ bevelled edge: against this the diaphragm of X-ray film, emulsion outwards, is held firmly by a screwed ring, so as to make it slightly convex and leaving about as small a dead space as is easily achievable. The inside of the tapered hole is polished longitudinally with rouge on a sliver of wood; turning marks are removed from the flat face of the chest-piece by scraping with a very carefully honed scraper, giving a sleeked mirror finish (the sleeks being less than a wavelength of light deep).

\section{Clinical Trials}

In clinical auscultation of the heart, the first model was found to behave much as had been expected. The higher frequencies were heard with much greater clarity than with an ordinary stethoscope. At the same time, the total volume of sound was rather low-presumably because of greater attenuation of extremely low frequencies (below 100 cycles). Several models were tried that differed in the size of the central hole, the slope of the tapered tube, and the angle of the bevel (which controls the convexity of the diaphragm and hence the volume of air behind it). We also tried different materials and thicknesses for the diaphragm.

Comparison of the performance of different stethoscopes presents certain difficulties. The power of the human auditory mechanism is such that once a sign has been detected with one model, the same observer may readily hear it with an inferior model. Judgement of the relative efficiency of two models may then be influenced by personal bias. In selecting the best model, we tried to avoid bias by having two, sometimes three, trained observers and arranging that the models for testing were identical in external appearance and were tried on ten patients with suitable physical signs.

As a result of these trials it was found that bakelite, $0.015 \mathrm{in}$. in thickness was superior to X-ray film for the diaphragm and that $\frac{1}{64}$ in. Tufnol (which is stiffer than bakelite) was better still. The angle of bevel that gave the smallest volume of air behind the diaphragm was the best. It was thought unwise to reduce the bevel angle below $18^{\circ}$.

It is sometimes said that the optimal tubing for stethoscopes should have an internal diameter of $\frac{1}{8}$ in. (Rappaport and Sprague, 1951). While this may possibly be true for low frequency sounds, it is certainly not true for high frequencies. The tubing of our first model was $\frac{1}{4}$ in. in internal diameter. In order to avoid the necessity of carrying two stethoscopes, we compromised by using $\frac{3}{16}$ in. metal ear tubes connected by 0.159 in. internal diameter polythene tubes to the bell chestpiece and the new diaphragm chest-piece which were both mounted on a $\frac{1}{4}$ in. two-way tap leading to a suitable Y-piece. In clinical use, the 0.159 in. tubing did not appear to impair the efficiency of the bell and this finding was substantiated by audiometer curves (the frequency of the audiometer only went down to 120 cycles). The new diaphragm appeared to be somewhat more sensitive with this arrangement. 
Mr. F. T. Bannister kindly made a facsimile of the first chest-piece for us and prepared engineering drawings (Fig. 1, 2, and 3). After verifying that the facsimile worked as well as the original when used with similar Y-piece and tubing, we made clinical trials of various combinations of Y-piece size, tubing diameter, and ear-tube diameter.
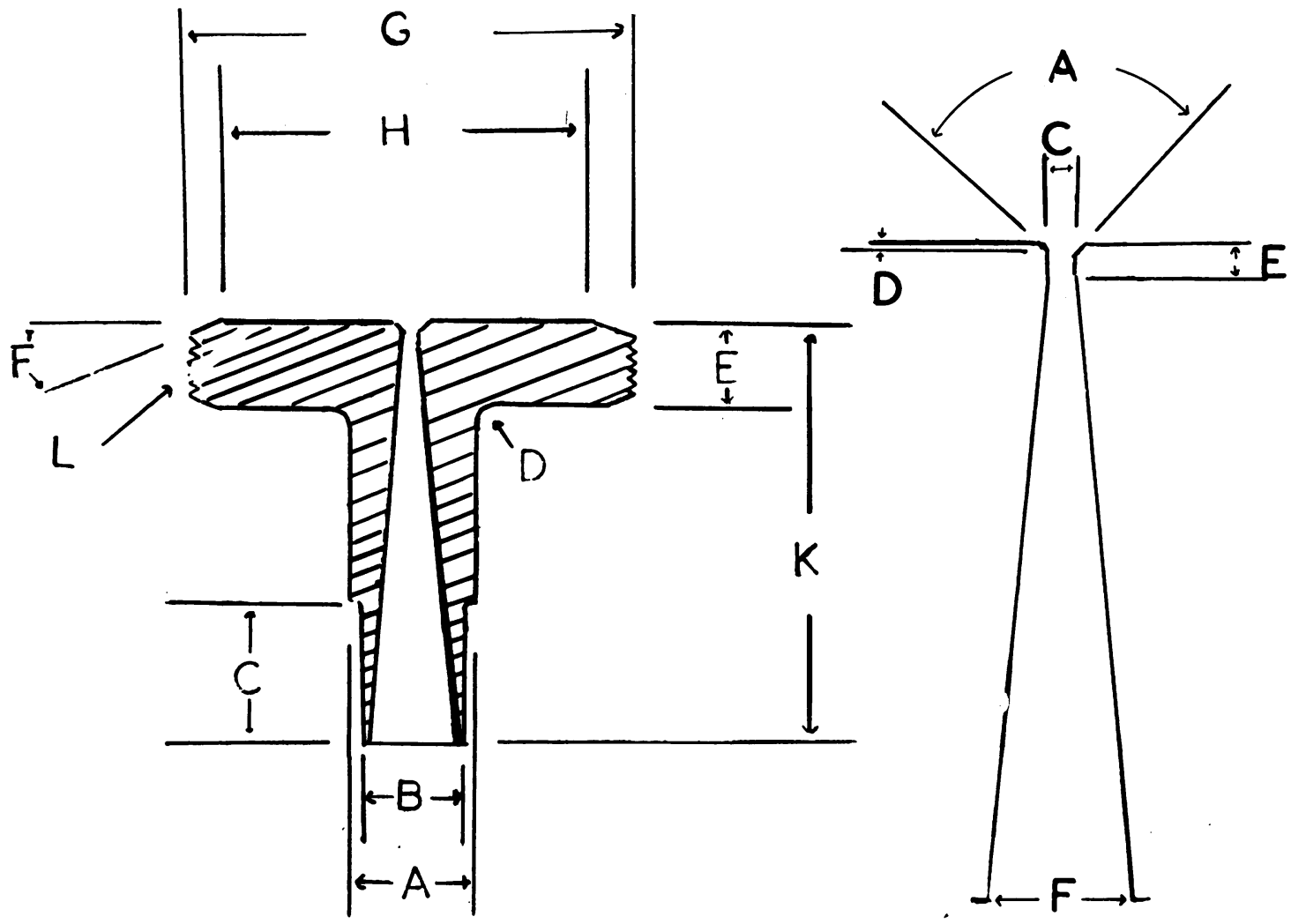

FIG. 1.-Cross-section of body of chest-piece. This is made of brass. $A=0.434$ in. diameter. $B=0.35$ in. diameter. $\quad C=15 / 32$ in. $\mathrm{D}=3 / 32$ in. radius. $\mathrm{E}=9 / 32$ in. $\mathrm{F}=18^{\circ} 10^{\prime} . \quad \mathrm{G}=1 \cdot 655$ in. diameter. $\mathrm{H}=1 \cdot 23 / 64$ in. diameter. $\mathrm{K}=1 \cdot 447$ in. $\mathrm{L}=40$ turns per inch thread.

The combination that we preferred has standard commercial $\frac{3}{16}$ in. ear tubes, $0 \cdot 159$ in. polythene tubes, 14 in. long, connected to a standard commercial Y-piece with a 2 -way tap, both of bore $\frac{1}{8}$ in. (giving a bell as an alternative). The tapered tube has been cut off $0.866 \mathrm{in}$. from the flat face and joined to the stub of $\frac{1}{8}$ in. bore tubing cut off a few millimeters from the spherical body of the tap. The diaphragm is of Tufnol*, $\frac{1}{64}$ in. thickness.

Preliminary measurements of the sensitivity of the combination of stethoscope and audiometer were made over frequencies ranging from 120 to 2000 cycles, using a standard audiometer crystal drive as the sound source, strapped to the under-side of the thigh just above the knee joint, while the

FIG. 2.-Details of bore of chest-piece. $A=90^{\circ} . \quad C=0.046$ in. $D=0.01$ in. $\mathrm{E}=0.06$ in. $\mathrm{F}=0.32$ in.

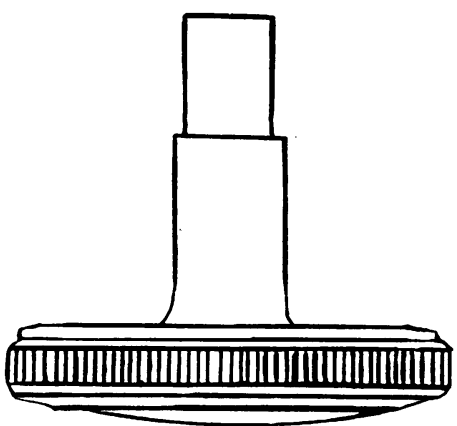

FIG. 3.-General external arrangement of chest-piece with Tufnol diaphragm clamped in position by screwed ring. Gap between diaphragm and stethoscope at centre 0.0315 in.

* Tufnol is bakelized linen fabric; it can be obtained from Tufnol, Ltd., Perry Barr, Birmingham. 
chest-piece was applied to the anterior surface of the thigh. These measurements express a property of the combination, and not a property of the stethoscope only; but the derived curves (Fig. 4 and 5), in which the ratio, sensitivity of new to sensitivity of standard stethoscope, was plotted against frequency, express a quantity nearly independent of the properties of the audiometer, but not necessarily independent of the physical characteristics of the observer's thigh, nor of the way he held

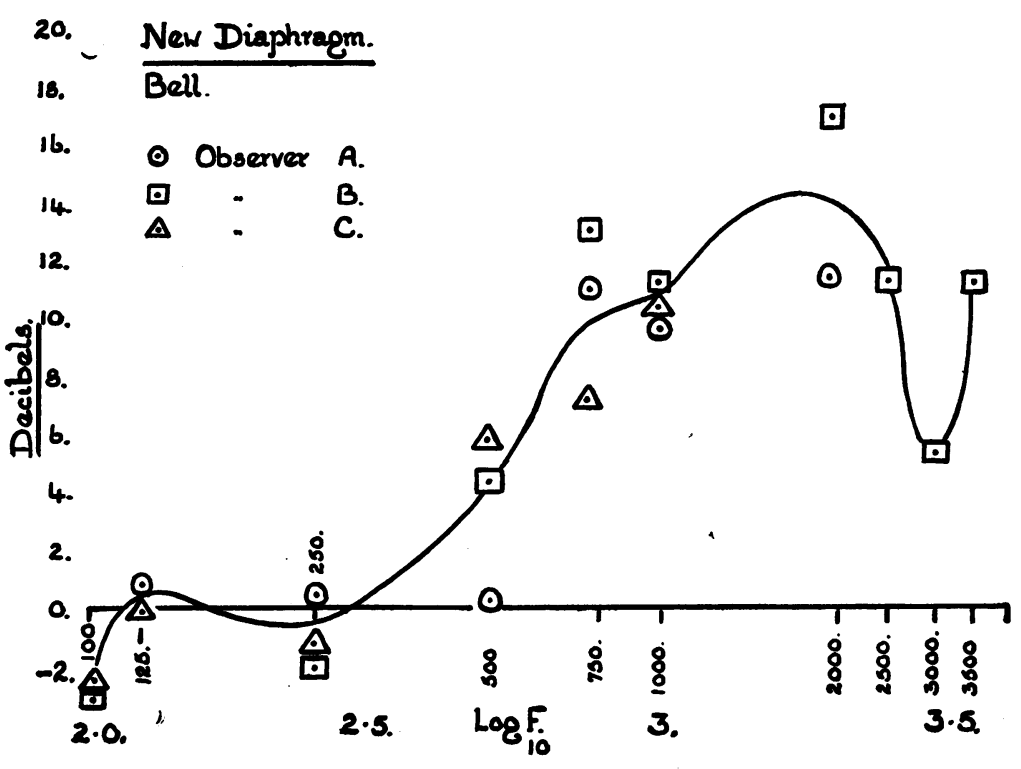

Fig. 4.-Audiometer curves showing the relative stethoscope sensitivities of the new diaphragm compared with the bell (three observers).

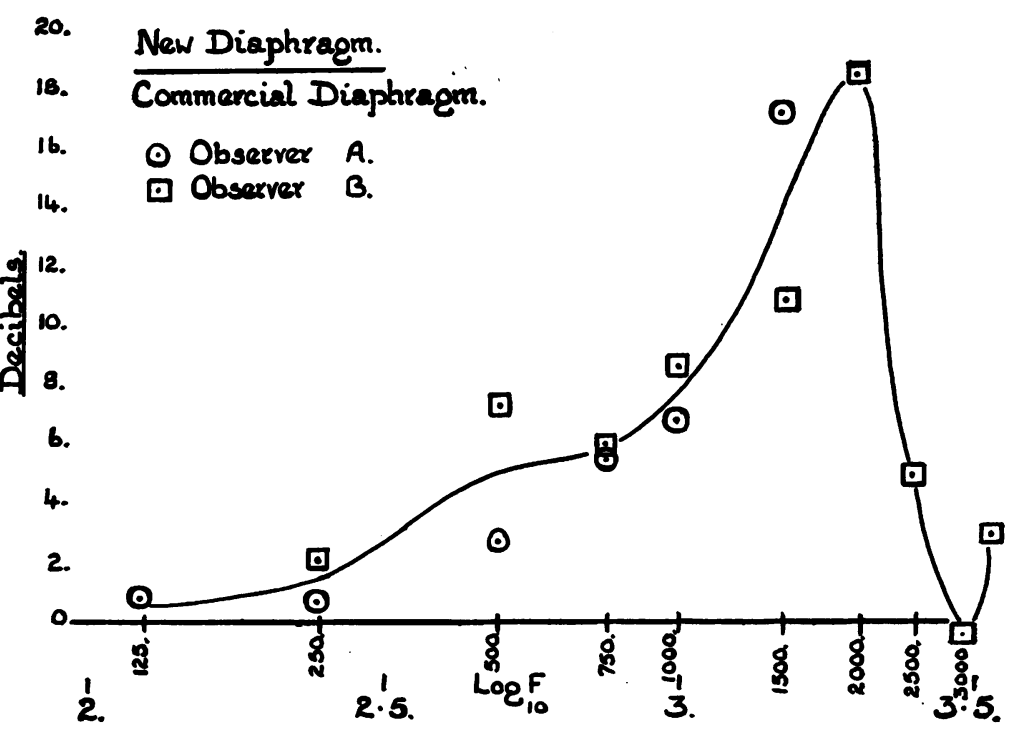

Fig. 5.-Audiometer curves showing the relative stethoscope sensitivities of the new diaphragm compared with the standard diaphragm stethoscope (two observers). 
the stethoscope against it. The peak sensitivity of the new stethoscope is seen to be at a substantially higher frequency than that of an average commercial diaphragm chest-piece. At extremely low frequencies, the bell is more sensitive than either. It is desirable, therefore, to mount a bell on a 2-way tap as an alternative.

The length of the tubing connecting the Y-piece to the head-piece was not found to be critical and 14 in. seemed convenient. A number of points about the diaphragm itself require emphasis. X-ray film has sometimes been recommended as suitable material (Wood, 1956) but we find it is too flexible. Sheet bakelite 0.015 in. thick is very much better. Unfortunately, manufacturers of stethoscopes use two different thicknesses of bakelite sheet, 0.015 in. and 0.009 in., and would seem to fit either indiscriminately. The $0.009 \mathrm{in}$. thick bakelite, like X-ray film, is too flexible. It is worth mentioning, moreover, that the suppliers may not possess micrometers, for on two occasions we have ordered bakelite diaphragms 0.015 in. thick and have received the thinner variety in a package labelled $0.015 \mathrm{in}$. If bakelite is used for the diaphragm, therefore, it is essential to verify that it is the correct thickness. It is perhaps not generally appreciated that the life of a bakelite diaphragm is limited. Once dented it loses its resilience and should be replaced. Perhaps more important is the liability of the diaphragm to insidious deterioration. In the majority of stethoscopes, when the diaphragm is clamped in position by the screwed ring, it is distorted with a convexity outwards. After a few weeks a bakelite diaphragm tends to assume a permanent "set" so that it is no longer under tension; worse still it may later develop a concave central depression. It is then found to have completely lost its resilience and to be quite ineffective. Sheet Tufnol ( $\left(\frac{1}{64}\right.$ in. thick) has proved a more suitable material. It attenuates low frequency sounds and has the advantage of distorting less easily than bakelite. We have found that the peak frequency sensitivity of the new stethoscope, when fitted with a Tufnol diaphragm, is much less readily altered by minor variations in the pressure with which the stethoscope is applied to the chest wall.

It is possible to make a reasonable approximation to the new stethoscope by filling the excess space behind the diaphragm of a commercial model with plasticine, reducing the diameter of the central hole to $1 \mathrm{~mm}$. This is most easily done in the common commercial model in which the diaphragm, when clamped by the screwed ring, becomes convex outwards. A piece of wire $1 \mathrm{~mm}$. in diameter (19 wire gauge) is held perpendicularly in the central hole of the chest-piece and the whole of the cavity is filled round it with plasticine. The wire is then withdrawn, leaving a $1 \mathrm{~mm}$. central hole. The surface of the plasticine should be flat and should be dusted with talcum powder to prevent it sticking to the diaphragm. When the diaphragm is clamped by the screwed ring its convexity leaves about the right air gap (Fig. 6). A stethoscope so treated will be found to attenuate low frequencies well and to have increased sensitivity in the higher frequency range. For listening to low frequency sounds, it will then of course be essential to have a bell as an alternative. A rough assessment of the efficiency of this model can be made by listening to a "noisy" heart murmur with the bell and diaphragm alternatively. If the diaphragm chest-piece is efficient, the frequency of the murmur should appear substantially higher, without reduction in volume, than when heard with the bell.

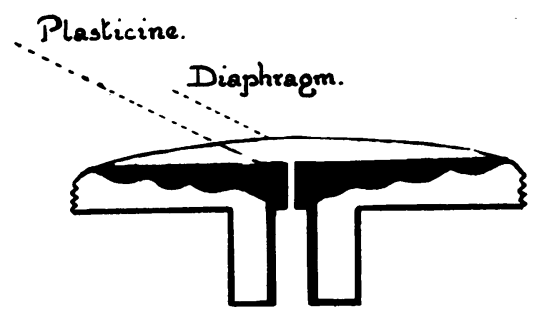

FIG. 6.-Cross-section of standard commercial diaphragm chest-piece with the excess scalloped space behind the diaphragm filled with plasticine to leave a $1 \mathrm{~mm}$. centre hole.

\section{Discussion}

We have already seen that the desirability of matching impedance at each of the junctionschest wall and diaphragm-unit, tube, Y-piece, and ear tubes, meatus, and auditory mechanism-led to the design of the first model in which we aimed at the best match possible at each junction assuming that the match at every other junction was perfect. This approach, though reasonable in a 
preliminary design, is a considerable over-simplification. It is not surprising, therefore, that some experimental modifications improve its performance in clinical application.

The stiffer diaphragm will reduce the sensitivity to extremely low frequencies, and over a certain range of frequencies may help to reduce the inevitable mismatching of impedance between chest wall and air. Polythene tubing stretches less easily than rubber; for this reason and also because it is smoother, we would expect its use to reduce acoustic damping. It may also have lower acoustic hysteresis. The degree of polish needed in acoustic systems may be judged by two main criteria. First, the mean free path of air molecules. If the surface irregularities measure less than this $\left(10^{-5} \mathrm{~cm}\right.$.) we may regard the polish as good enough; since this is nearly smooth enough to make a good mirror. Secondly, a less severe criterion is the shear wave attenuation distance $\sqrt{\frac{\nu \mathrm{cm}}{P}}$, where $\nu=$ kinematic viscosity of air, i.e. viscosity/density $\left(0 \cdot 143 \mathrm{~cm} .{ }^{2} \mathrm{sec}^{-1}\right)$, and $P$ is $2 \pi \times$ frequency. This is (roughly) the thickness of the more or less stagnant layer of air near the walls of the device: it is about $0.05 \mathrm{~mm}$. for 1000 cycles and $0.15 \mathrm{~mm}$. for 100 cycles. Hence it is not a negligible fraction of the space behind the diaphragm and also of the radius of the $1 \mathrm{~mm}$. hole. Surface irregularities that are comparable with the shear wave attenuation distance are presumably too big. This is why we regard a fairly good turned finish as inadequate.

The Tapered Tube. We have examined the rather heavy algebraic formulæ that express (when viscosity is neglected) the admittance of a tapered tube connected to a parallel tube terminated in a reflection free manner. The tapered tube does not depart much in behaviour from a simple series inductance till it is a wavelength or more long. It does not form an impedance transformer, whereby a narrow tube can be joined with negligible reflection to a wide one, until both the tapered length and the distance to the vertex are many wavelengths long. We made the inductance of our tapered tube to suit the chest-piece for the desired mode of resonance. (The term "venturi" for this tube is perhaps best avoided. The physics of steady flow is profoundly different from that of low amplitude sound waves and the reason for which one uses a venturi in steady flow-to avoid turbulence-does not arise in acoustics.)

It is convenient in calculating the resonance to transform the acoustic quantities into their mathematical equivalents in the electrical analogue circuit so that the more familiar electrical formulæ may be used. This is done in the appendix (p.454), where it is shown that a resonance frequency around 1000 cycles may be expected and that the resonance will be rather broad.

\section{Clinical Applications}

The increased sensitivity of the new stethoscope to high frequency sounds and its selective attenuation of the lower frequencies have useful applications in clinical auscultation. The majority of ejection and regurgitant systolic murmurs and mid-diastolic murmurs have their maximal amplitude in the low frequency range. Such murmurs are therefore best heard with a bell chest-piece. Soft mitral or tricuspid regurgitant murmurs, however, often have a higher frequency and are then much more readily heard with the new stethoscope which brings out clearly their late systolic crescendo. With a bell stethoscope it is sometimes difficult to detect the gap between the end of a mid-systolic murmur and the second sound. The attenuation of low frequency components of the murmur by the new stethoscope often enables the mid-systolic timing of the murmur to be more clearly identified.

The early diastolic murmurs of aortic and pulmonary regurgitation are generally in the higher frequency range, especially when they are faint. On several occasions the new stethoscope has enabled us to hear such murmurs at a time when they were inaudible with standard diaphragm chest-pieces.

The main clinical usefulness of the new diaphragm, however, lies in the clarity with which it enables the high frequency components of normal and abnormal heart sounds to be heard. Asynchronous closure of atrio-ventricular or semilunar valves may be recognized clinically when the sounds 
of valve closure are heard to be separated or split (Leatham, 1954). The sound of valve closure consists of high frequency vibrations of brief duration spanned by longer lasting low frequency components. Wide splitting of heart sounds is readily detected, but when the split is narrow it tends to be obscured by overlapping of the low frequency components. With the new stethoscope, the low frequency sounds are weakened and the much briefer high frequency components are audible as sharp clear sounds. Very narrow splitting can thus be appreciated. Indeed, we have noted that in many normal subjects the second heart sound remains narrowly split even at the end of expiration, whereas with a standard diaphragm stethoscope it appears to be single. In many patients with systolic hypertension or ischæmic heart disease, we have been able to hear "crossed" or "biphasic" splitting of the second heart sound during respiration; i.e. a narrowly split second sound at the end of expiration is heard to become single in mid-inspiration and to split again at the end of inspiration, the reverse taking place during the expiratory phase. This presumably indicates slight prolongation of left ventricular ejection with consequent delay in aortic valve closure. Thus, at the end of expiration, the aortic valve closes just after the pulmonary valve. During inspiration the normal progressive prolongation of right ventricular ejection leads to pulmonary valve closure first coinciding with aortic valve closure, and then following after it. The pulmonary element of the second sound migrates in the opposite direction during the expiratory phase. The resulting splits are necessarily narrow and we have found great difficulty in detecting them with an ordinary stethoscope.

The stethoscope is particularly useful in detecting normal and abnormal splitting of heart sounds in the presence of low frequency murmurs. Paradoxical splitting of the second heart sound (Gray, 1956) may be difficult to hear in aortic stenosis if the murmur spans the pulmonary element. A similar difficulty is commonly encountered in persistent ductus arteriosus. The attenuation of low frequencies by the new diaphragm facilitates detection of the sign.

The wide difference in frequency sensitivity between the bell and the diaphragm chest-piece is sometimes of considerable help in deciding the nature of a particular heart sound. For example, in mixed mitral stenosis and regurgitation, it is sometimes difficult to differentiate between an opening snap and a third sound at the apex. Since the latter is a low frequency sound, it is heard much more readily with the bell and may be inaudible with the diaphragm; the reverse, of course, is true of an opening snap. Differentiation between an atrial gallop sound and physiological splitting of the first sound can often be made by comparing the audibility of the sound with the bell and the diaphragm chest-piece.

It is true that the human auditory mechanism has considerable power of selection, a facility that can be appreciably improved by practice. Indeed, a skilled auscultator may claim that most of the signs we have mentioned can be heard with a simple bell stethoscope. It would, however, seem as illogical an aim to acquire auscultatory virtuosity with an insensitive stethoscope as to learn to palpate the abdomen with the gloved hand.

\section{SUMmaRY}

The frequency components of heart sounds and murmurs between approximately 250 and 1500 cycles appear to be of clinical importance. Standard commercial diaphragm stethoscopes are not designed for high sensitivity in this range. A new diaphragmatic stethoscope was therefore designed to have its maximum sensitivity around 1000 cycles. Electrical analogue formulæ were used to predict the frequency of maximum response. Audiometer curves confirm that the new stethoscope is more sensitive in this frequency range than standard models.

In clinical auscultation, the new stethoscope enables faint high frequency murmurs to be heard much more readily; it greatly facilitates the detection of narrow splitting of heart sounds.

We present this work in the hope of inducing others with more time and facilities to make a more searching investigation of the ideal design for a stethoscope. 
Gray, I. (1956). Brit. Heart J., 18, 1.

\section{REFERENCES}

Leatham, A. (1954). Lancet., 2, 607.

Rappaport, M. B., and Sprague, H. B. (1951). Amer. Heart J., 42, 605.

Wood, P. (1956). Diseases of the Heart and Circulation, p. 60.

\section{APPENDIX}

The Resonance-First Mode. For convenience in calculating the resonance, we may transform the acoustic quantities into their mathematical equivalents in the electrical analogue circuit so that the more familiar electrical formulæ can be used. An acoustic pressure of 1 dyne $/ \mathrm{cm}^{2}$ then becomes a "pseudo-volt" or " $\psi$-volt" and an acoustic flow of $1 \mathrm{ml}$./sec. becomes a "pseudo-amp" or " $\psi$-amp." (A " $\psi$-watt" denotes an acoustic power of 1 erg./sec., i.e. $0 \cdot 1$ microwatt.)

In the frequency range to which the ear is most sensitive, we may suppose as a first approximation that reflection at the auditory meatus may be neglected. If we neglect viscosity the stethoscope tube, radius $r$, is equivalent to a loss-free transmission line of inductance per $\mathrm{cm}$. length (inertial effect) $\rho / \pi r^{2} \mathrm{gram} . \mathrm{cm}^{-5}$ $\left(=L_{1}\right)$ where $\rho$ is the density of air, together with capacity per $\mathrm{cm}$. length (cushioning effect) $\pi r^{2} / \gamma P$ gram. ${ }^{-1} \mathrm{sec}^{2}{ }^{2} \mathrm{~cm} .{ }^{3}\left(=C_{1}\right)$, where $P$ is the atmospheric pressure and $\gamma$ is the ratio of the specific heats of air $\left(c_{p} / c_{v}\right)$. Accordingly when the tube is terminated without reflection. its input impedance will be purely resistive and of amount $R \doteqdot \sqrt{\frac{L_{1}}{C_{1}}}$ i.e. $\frac{1}{\pi r^{2}} \sqrt{\frac{\rho}{\gamma P}}$ or $\frac{\gamma P}{a \pi r^{2}}$ gram.cm..$^{-4} \mathrm{sec}^{-1}$ (" $\psi$-ohms")-where $a$ is the velocity of sound. That is, $R=\frac{13 \cdot 4}{r^{2}} \psi$-ohms. The inductance $L$ of the tapered tube, radii $r_{1}, r_{2}$, length $l$ is $\frac{\rho l}{\pi r_{1} r_{2}}$ gram cm. ${ }^{-4}$ i.e. $L \doteqdot \frac{3 \cdot 82 \times 10^{-4} l}{r_{1} r_{2}}$ “ $\psi$-henries."

The capacity $C$ of a dead space volume $V \mathrm{ml}$. is $\frac{V}{\gamma P}$ gram $^{-1} \mathrm{sec}^{2}{ }^{2} \mathrm{~cm} .{ }^{4}$ i.e. $C=7 \times 10^{-7}$ " $\psi$-farads."

For our system $r_{1} \doteqdot 0.05 \mathrm{~cm}$., $r_{2} \doteqdot 0.3 \mathrm{~cm}$.; $V \doteqdot \frac{1}{2} \mathrm{ml}$. We shall have therefore

Resistance, $R=150 \psi$-ohms,

Inductance, $L=0.064 \psi$-henries, Capacity, $C=3.5 \times 10^{-7} \psi$-farads.

There will be parallel circuit resonance when $1-p^{2} L C=\frac{C R^{2}}{L} \doteqdot \frac{1}{8}$ and this will occur when $p=2 f=6681$, giving a resonant frequency $f$ of 1063 cycles/sec. The large value, $\frac{1}{8}$ of $\frac{C R^{2}}{L}$, shows that the resonance will be a broad one. The calculations should be regarded as giving orders of magnitude only because the estimation of dead space is only rough (the dead space changes appreciably when the chest-piece is pressed lightly against the chest), no correction has been made for viscosity, and, most important, the auditory meatus will not form a strictly reflection-free termination for the ear tube.

The formulæ help to explain why the use of a $\frac{1}{8}$ in. Y-piece instead of $\frac{1}{4}$ in. improves the clinical performance. It will lower the resonant frequency and the content of heart-sound above 1000 cycles $/ \mathrm{sec}$. is relatively small. A tapered tube $\frac{3}{4} \mathrm{~mm}$. instead of $1 \mathrm{~mm}$. diameter seemed slightly worse.

The Resonance-Second Mode. At low frequencies the auditory meatus cannot form a reflection-free termination for the ear tubes (assuming the drum is imperforate). Thus its impedance must be principally capacitative. The preceding considerations will then cease to apply and there may be a second mode of resonance in which we have the capacity (and equivalent series resistance) of the meatus, the capacity of the chest-piece, the inductance of the tapered tube, and the inductance (with some distributed capacity) of the ear tubes, all in series. This is presumably the mode of resonance exploited in one variety of twin chestpiece stethoscope, both the bell and, to some extent, the diaphragm having larger capacities than the chestpiece that we describe, and being ordinarily used with narrower ear tubes $(0.118$ in. internal diameter measured; $0 \cdot 125$ in. nominal) - so that a resonant frequency may occur at a value low enough for the ear to behave capacitatively, as postulated in the second mode. The length of the ear tubes may be important in this mode, because it controls the resonant frequency.

In the first mode, on the other hand, with little reflection at the meatus, the length of the ear tubes is unimportant. 